\title{
Bounds on convection driven by internal heating
}

$\mathrm{Lu} \mathrm{Lu}$

Department of Mathematics, University of Michigan, Ann Arbor, Michigan 48109-1109

Charles R. Doering

Department of Mathematics and Michigan Center for Theoretical Physics, University of Michigan, Ann Arbor, Michigan 48109-1109

Friedrich H. Busse

Theoretical Physics IV, University of Bayreuth, 95440 Bayreuth, Germany

(Received 20 January 2004; accepted 1 April 2004; published online 14 June 2004)

Bounds are derived for the space-time averaged temperature $\langle T\rangle$ of a fluid layer in the Boussinesq approximation between fixed-temperature horizontal boundaries subject to uniform heating $H$ throughout the volume. The analysis is carried out for both finite and infinite Prandtl number fluids. While the average temperature $\langle T\rangle$ $\sim H$ in the purely conductive state, convection enhances the heat transport beyond static conduction reducing the temperature. Lower bounds to the average temperature of the layer scale with the magnitude of the imposed heat flux, with one scaling exponent for the arbitrary Prandtl number case and another for the infinite Prandtl number model. Specifically, it is proven here that at large heating rates where convection is important, $\langle T\rangle \geqslant c_{1} H^{2 / 3}$ for finite Prandtl number fluids and $\langle T\rangle$ $\geqslant c_{2} H^{5 / 7}$ for infinite Prandtl number fluids. Explicit prefactors $c_{1}$ and $c_{2}$ for the scaling bounds are computed as well. (c) 2004 American Institute of Physics.

[DOI: $10.1063 / 1.1760845$ ]

\section{INTRODUCTION}

Turbulent transport of mass, momentum, and heat remains one of the most important problems for modern theoretical physics and applied mathematics. For incompressible fluid flows, fundamental models such as the Navier-Stokes and related equations are believed to quantitatively describe these phenomena. However the complexity of the dynamics in these systems of nonlinear partial differential equations prohibits exact solutions, and the wide range of length and time scales in turbulent solutions makes direct numerical simulation extremely challenging and expensive. One mathematical approach to the analysis of these systems is to derive rigorous bounds on physically relevant quantities. ${ }^{12,3,8}$ This approach is of more than just mathematical interest because it turns out that in some cases the bounds tend to capture aspects of the turbulent scaling of the quantities with respect to the control parameters (e.g., the Reynolds or Rayleigh number). In the case of Rayleigh-Bénard convection, for example, where a fluid layer between horizontal plates is heated from below, the enhancement of the heat flux due to convection, usually measured by a Nusselt number, can be bounded from above in terms of the temperature drop across the layer ${ }^{11,1,9,6,10,14}$ expressed in terms of the Rayleigh number.

In this paper we consider the problem of convective heat transport in a fluid layer between fixed-temperature horizontal boundaries with uniform heating throughout the volume. This problem is motivated by geophysical applications; $;{ }^{7,17}$ the Earth's plate tectonics is a result of convection in the mantle which is predominantly driven by uniform heating due to radioactive decay of elements distributed throughout the mantle. Mantle dynamics is generally modeled as the flow of a high (infinite) Prandtl number fluid with strongly temperature dependent viscosity. The models we focus on here are simpler, with constant material parameters. The boundary conditions for mantle convection are complicated-especially on "top"—but we restrict the investigation here to rigid no-slip isothermal boundaries in order to make progress. In principle, if all the relevant 
materical and boundary effects could be included, the kinds of bounds derived here could be used to put limits on the thermal history of the Earth. A distinct engineering application of this kind of analysis is to the problem of nuclear reactor meltdown. ${ }^{4}$

The rest of this paper is organized as follows: in the next section we present the details of the models we will analyze. In a brief Sec. III we apply the "background" method ${ }^{9}$ to the arbitrary Prandtl number problem to derive a scaling lower bound on the space-time averaged temperature of the layer along with an explicit prefactor. In the following Sec. IV we apply a multiple boundary layer asymptotic theory ${ }^{1}$ to sharpen the estimate, increasing the prefactor in the lower bound by a factor of 4 . Section V is concerned with the infinite Prandtl number problem, and the background method utilizing a recently derived inequality ${ }^{10}$ results in a scaling lower bound with a smaller exponent. In the concluding Sec. VI we summarize our results in the context of direct numerical simulations, and discuss some possible areas for further development of this approach.

\section{GOVERNING EQUATIONS}

The fluid layer is confined between two parallel plates of horizontal extent $L_{x}$ and $L_{y}$ separated by vertical $(z)$ distance $d$. The no-slip upper and lower plates are held at fixed temperatures $T_{0}$ and $T_{1}$, respectively; the temperature difference $\Delta T=T_{0}-T_{1}$ which will eventually be taken to be zero for the work presented here. A uniform volumetric heat flux $H$ (with units power/ volume) is pumped into the layer. The governing equations for the velocity field $\mathbf{u}$, the pressure $p$ and the temperature $T$ in the standard Boussinesq approximation are

$$
\begin{gathered}
\frac{\partial \mathbf{u}}{\partial t}+\mathbf{u} \cdot \nabla \mathbf{u}=-\nabla p+\nu \nabla^{2} \mathbf{u}+\hat{\mathbf{k}} g \alpha T, \\
\frac{\partial T}{\partial t}+\mathbf{u} \cdot \nabla T=\kappa \nabla^{2} T+\gamma, \\
\nabla \cdot \mathbf{u}=0,
\end{gathered}
$$

with the boundary conditions

$$
\left.\mathbf{u}\right|_{z=0, d}=0,\left.\quad T\right|_{z=0}=T_{0},\left.\quad T\right|_{z=d}=T_{1},
$$

where $\nu$ is the viscosity, $g$ is the acceleration of gravity along the $z$ axis (in the $-\hat{\mathbf{k}}$ direction), $\alpha$ is the thermal expansion coefficient, $\kappa$ is the thermal diffusion coefficient and $\gamma=H / \rho c$, where $\rho$ is the density and $c$ is the specific heat capacity of the fluid. We impose periodic boundary conditions in the horizontal directions with periods $L_{x}$ and $L_{y}$.

Using $d^{2} / \kappa$ as the unit of time, $d$ as the unit of length, and $\gamma d^{2} / \kappa$ as the unit of temperature, the governing equations are put into the nondimensional form

$$
\begin{gathered}
\operatorname{Pr}^{-1}\left(\frac{\partial \mathbf{u}}{\partial t}+\mathbf{u} \cdot \nabla \mathbf{u}\right)+\nabla p=\nabla^{2} \mathbf{u}+R T \hat{\mathbf{k}}, \\
\frac{\partial T}{\partial t}+\mathbf{u} \cdot \nabla T=\nabla^{2} T+1,
\end{gathered}
$$

where $\operatorname{Pr}=\nu / \kappa$ is the Prandtl number and $R=g \alpha d^{5} \gamma / \kappa^{2} \nu$ is the heat Rayleigh number. ${ }^{16}$ We consider $R$, proportional to the internal heating rate, to be the control parameter. The boundary conditions in nondimensional form are

$$
\left.\mathbf{u}\right|_{z=0,1}=0,\left.\quad T\right|_{z=0}=\widetilde{T},\left.\quad T\right|_{z=1}=0,
$$


where $\widetilde{T}=\left(\kappa / \gamma d^{2}\right) \Delta T$; this shows that $R \widetilde{T}$ is the usual Rayleigh number $\mathrm{Ra}=g \alpha \Delta T d^{3} / \nu \kappa$ for bottom heating.

In the following discussion, we only consider the special case where both boundaries are held at the same temperature, i.e., $\Delta T=0$ or $\widetilde{T}=0$. With this boundary condition, the static conduction solution has a quadratic profile:

$$
T=\frac{1}{2} z(1-z),
$$

which becomes unstable for sufficiently large $R .{ }^{19}$ Once convection sets in, the flow tends to lower the average temperature of the fluid, so the estimate of interest is the minimum possible bulk average temperature for a given value $R$. We define the space-time average of a function $f(\mathbf{x}, t)$ as

$$
\langle f\rangle=\lim _{t \rightarrow \infty} \frac{1}{t} \int_{0}^{t} d s \frac{d^{2}}{L_{x} L_{y}} \int d x d y d z f(\mathbf{x}, s) .
$$

In the following discussion, we apply the background and multiple boundary layer methods to derive lower bounds for the bulk average (nondimensional) temperature with respect to $R$ in the form $\langle T\rangle \geqslant c R^{\alpha}$ as $R \rightarrow \infty$.

\section{BACKGROUND METHOD FOR FINITE Pr}

To apply the background method, first we decompose the temperature field $T(x, y, z, t)$ into a time-independent background profile $\tau(z)$ and a fluctuating part $\theta(x, y, z, t)$ :

$$
T(x, y, z, t)=\tau(z)+\theta(x, y, z, t) .
$$

The boundary conditions of $T(x, y, z, t)$ are contained in $\tau(z)$ :

$$
\tau(0)=\tau(1)=0
$$

and the fluctuating part $\theta(x, y, z, t)$ satisfies homogeneous boundary conditions

$$
\theta(x, y, 0, t)=\theta(x, y, 1, t)=0 .
$$

The velocity field $\mathbf{u}$ is divergence-free with no-slip boundary conditions:

$$
\nabla \cdot \mathbf{u}=0,\left.\quad \mathbf{u}\right|_{z=0,1}=0 .
$$

With this decomposition the governing Eqs. (2) become

$$
\begin{gathered}
\operatorname{Pr}^{-1}\left(\frac{\partial \mathbf{u}}{\partial t}+\mathbf{u} \cdot \nabla \mathbf{u}\right)+\nabla p=\nabla^{2} \mathbf{u}+\tau \hat{\mathbf{k}}+R \theta \hat{\mathbf{k}}, \\
\frac{\partial \theta}{\partial t}+\mathbf{u} \cdot \nabla \theta=\nabla^{2} \theta+\tau^{\prime \prime}+1-w \tau^{\prime}
\end{gathered}
$$

Then taking the space-time average of $\mathbf{u} \cdot(9 \mathrm{a})$ yields

$$
\left\langle|\nabla \mathbf{u}|^{2}\right\rangle=R\langle w \theta\rangle,
$$

and averaging $\theta \cdot(9 \mathrm{~b})$ and $\tau \cdot(9 \mathrm{~b})$ yield, respectively,

$$
\begin{gathered}
\left\langle w \theta \tau^{\prime}\right\rangle=-\left\langle|\nabla \theta|^{2}\right\rangle-\left\langle\theta_{z} \tau^{\prime}\right\rangle+\langle\theta\rangle, \\
-\left\langle w \theta \tau^{\prime}\right\rangle=-\left\langle\theta_{z} \tau^{\prime}\right\rangle+\langle\tau\rangle-\left\langle\tau^{\prime 2}\right\rangle .
\end{gathered}
$$


The difference of the above two identities is

$$
(\langle\theta\rangle-\langle\tau\rangle)=\left\langle|\nabla \theta|^{2}\right\rangle+2\left\langle w \theta \tau^{\prime}\right\rangle-\left\langle\tau^{\prime 2}\right\rangle .
$$

Since $\langle T\rangle=\langle\tau\rangle+\langle\theta\rangle$, we have

$$
\langle T\rangle=\left\langle|\nabla \theta|^{2}\right\rangle+2\left\langle w \theta \tau^{\prime}\right\rangle+2\langle\tau\rangle-\left\langle\tau^{\prime 2}\right\rangle .
$$

The identity (10) can also be written as

$$
0=\frac{a}{R}\left\langle|\nabla \mathbf{u}|^{2}\right\rangle-a\langle w \theta\rangle,
$$

where $a$ is a positive number (a "balance parameter") to be adjusted to yield the best prefactor. ${ }^{13}$ Adding Eq. (15) to Eq. (14) enables us to express the average temperature as follows:

$$
\langle T\rangle=2\langle\tau\rangle-\left\langle\tau^{\prime 2}\right\rangle+H,
$$

where

$$
H=\left\langle|\nabla \theta|^{2}\right\rangle+\left\langle\left(2 \tau^{\prime}-a\right) w \theta\right\rangle+\frac{a}{R}\left\langle|\nabla \mathbf{u}|^{2}\right\rangle .
$$

If the functional $H$ is positive semidefinite among the fields $\mathbf{u}$ and $\theta$ satisfying

$$
\nabla \cdot \mathbf{u}=0,\left.\quad \mathbf{u}\right|_{z=0,1}=0,\left.\quad \theta\right|_{z=0,1}=0,
$$

then we have a lower bound for $\langle T\rangle$ :

$$
\langle T\rangle \geqslant 2\langle\tau\rangle-\left\langle\tau^{\prime 2}\right\rangle .
$$

So the goal is to choose a background profile $\tau$ satisfying the boundary conditions (6) guaranteeing that $H$ is positive semidefinite while making the lower bound in (18) as large as possible.

If we could take a linear background profile with the slope $a / 2>0$, then $2 \tau^{\prime}-a$ would vanish and thus the functional $H$ would clearly be non-negative, but this choice can not allow both $\tau(0)$ and $\tau(1)$ to vanish simultaneously. However, the indefinite term in $H$ is proportional to $w \theta$ that vanishes at the boundaries. This suggests that we can take $2 \tau^{\prime}=a$ in the middle while introducing two boundary layers to enforce $\tau$ 's boundary conditions. These considerations lead us to focus on the family of piecewise linear background profiles

$$
\tau(z)=\left\{\begin{array}{l}
\left(\frac{a}{2}+\frac{b}{\delta_{1}}\right) z, \quad 0 \leqslant z<\delta_{1}, \\
\frac{a}{2} z+b, \quad 1-\delta_{1} \leqslant z \leqslant 1-\delta_{2}, \\
-\left[\frac{a}{2}\left(\frac{a}{\delta_{2}}-1\right)+\frac{b}{\delta_{2}}\right](z-1), \quad 1-\delta_{2} \leqslant z \leqslant 1,
\end{array}\right.
$$

where $\delta_{1}\left(\delta_{2}\right)$ is the thickness of the boundary layer at $z=0(z=1)$ introduced to satisfy the boundary conditions (see Fig. 1). Then

$$
\langle T\rangle \geqslant 2\langle\tau\rangle-\left\langle\tau^{\prime 2}\right\rangle=\frac{a}{2}\left(1-\delta_{2}\right)+b\left(1-\delta_{1}-\delta_{2}\right)-\left\{\frac{a^{2}}{4}\left(\frac{1}{\delta_{2}}-1\right)+b^{2}\left(\frac{1}{\delta_{1}}+\frac{1}{\delta_{2}}\right)+\frac{a b}{\delta_{2}}\right\},
$$

provided the quadratic functional $H$ in (17) is positive definite. Before estimating the size of $H$, we can maximize $2\langle\tau\rangle-\left\langle\tau^{\prime 2}\right\rangle$ over $a$ and $b$, and this procedure yields 


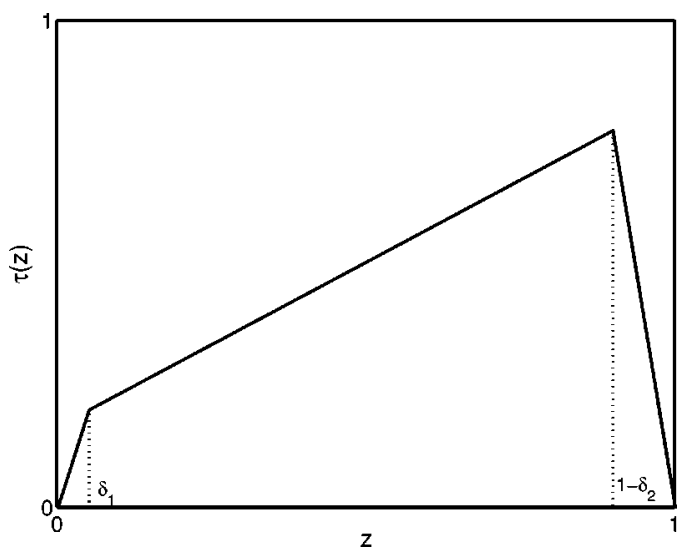

FIG. 1. The background profile for finite Prandtl number.

$$
\langle T\rangle \geqslant \frac{1}{4}\left(1-\delta_{1}\right)\left(1-\delta_{2}\right)\left(\delta_{1}+\delta_{2}\right)
$$

with

$$
\left\{\begin{array}{l}
a=\delta_{2}-\delta_{1} \\
b=\frac{\delta_{1}}{2}\left(1-\delta_{2}\right) .
\end{array}\right.
$$

Then using the inequality ${ }^{9}$

$$
\left|\int_{0}^{1} w \theta\right| \leqslant\left(\frac{c}{4}\|\mathbf{u}\|_{2}^{2}+\frac{1}{c}\|\theta\|_{2}^{2}\right)
$$

for any $c>0$, the term $\left\langle\left(2 \tau^{\prime}-a\right) w \theta\right\rangle$ can be estimated by

$$
\begin{aligned}
\left|\left\langle\left(2 \tau^{\prime}-a\right) w \theta\right\rangle\right| & \leqslant \frac{b \delta_{1}}{2}\left[\frac{c_{1}}{4}\left\langle|\nabla \mathbf{u}|^{2}\right\rangle+\frac{1}{c_{1}}\left\langle|\nabla \theta|^{2}\right\rangle\right]+\frac{(a+2 b) \delta_{2}}{4}\left[\frac{c_{2}}{4}\left\langle|\nabla \mathbf{u}|^{2}\right\rangle+\frac{1}{c_{2}}\left\langle|\nabla \theta|^{2}\right\rangle\right] \\
& =\frac{\delta_{1}^{2}\left(1-\delta_{2}\right)}{4}\left[\frac{c_{1}}{4}\left\langle|\nabla \mathbf{u}|^{2}\right\rangle+\frac{1}{c_{1}}\left\langle|\nabla \theta|^{2}\right\rangle\right]+\frac{\delta_{2}^{2}\left(1-\delta_{1}\right)}{4}\left[\frac{c_{2}}{4}\left\langle|\nabla \mathbf{u}|^{2}\right\rangle+\frac{1}{c_{2}}\left\langle|\nabla \theta|^{2}\right\rangle\right] .
\end{aligned}
$$

Then

$$
H \geqslant\left[1-\frac{\delta_{1}^{2}\left(1-\delta_{2}\right)}{4 c_{1}}-\frac{\delta_{2}^{2}\left(1-\delta_{1}\right)}{4 c_{2}}\right]\left\langle|\nabla \theta|^{2}\right\rangle+\left[\frac{a}{R}-\frac{\delta_{1}^{2}\left(1-\delta_{2}\right) c_{1}}{16}-\frac{\delta_{2}^{2}\left(1-\delta_{1}\right) c_{2}}{16}\right]\left\langle|\nabla \mathbf{u}|^{2}\right\rangle .
$$

Thus $H$ is positive semidefinite if

$$
1-\frac{\delta_{1}^{2}\left(1-\delta_{2}\right)}{4 c_{1}}-\frac{\delta_{2}^{2}\left(1-\delta_{1}\right)}{4 c_{2}} \geqslant 0
$$

and

$$
\frac{a}{R}-\frac{\delta_{1}^{2}\left(1-\delta_{2}\right) c_{1}}{16}-\frac{\delta_{2}^{2}\left(1-\delta_{1}\right) c_{2}}{16} \geqslant 0 .
$$

We can choose $c_{1}=c_{2}=c$ and then it is sufficient to require 


$$
\left[\delta_{1}^{2}\left(1-\delta_{2}\right)+\delta_{2}^{2}\left(1-\delta_{1}\right)\right]^{2}=\frac{64\left(\delta_{2}-\delta_{1}\right)}{R},
$$

and

$$
c=\frac{a}{4}
$$

Now the lower bound of $\langle T\rangle$ in (21) can be maximized over $\delta_{1}$ and $\delta_{2}$ subject to condition (26). But before fully optimizing the bound in (21) we consider the special case where there is only one boundary layer in the background field at $z=1$, i.e., the choice $\delta_{1}=0$. Although this will not give us the optimal bound, it is still a rigorous lower bound which is easier to compute and which can be compared with the optimal bound later.

For $\delta_{1}=0$ we should set $b=0$ in the general background profile (19). Thus (21) becomes

$$
\langle T\rangle \geqslant \frac{1}{4}\left(1-\delta_{2}\right) \delta_{2},
$$

and the constraint (26) is simplified to be

$$
\delta_{2}^{3}=\frac{64}{R}
$$

We can now write down the estimate

$$
\langle T\rangle \geqslant R^{-1 / 3}\left(1-4 R^{-1 / 3}\right)
$$

So as $R \rightarrow \infty,\langle T\rangle \geqslant R^{-1 / 3}$ with prefactor 1 .

To fully optimize the bound, we need to maximize the right-hand side of the inequality (21) subject to the constraint (26):

$$
\langle T\rangle \geqslant \max _{\delta_{1}, \delta_{2}}\left\{\frac{1}{4}\left(1-\delta_{1}\right)\left(1-\delta_{2}\right)\left(\delta_{1}+\delta_{2}\right)\right\},
$$

with $\delta_{1}$ and $\delta_{2}$ satisfying

$$
\left[\delta_{1}^{2}\left(1-\delta_{2}\right)+\delta_{2}^{2}\left(1-\delta_{1}\right)\right]^{2}=\frac{64\left(\delta_{2}-\delta_{1}\right)}{R} .
$$

This is easily done numerically and the result is shown in Fig. 2. It is seen from the graph that this better bound follows the same scaling as in (30), i.e., $\sim R^{-1 / 3}$ as $R \rightarrow \infty$. The prefactor can be measured from the graph, showing that the asymptotic prefactor is improved slightly:

$$
\langle T\rangle \geqslant 1.09 R^{-1 / 3} \text { as } R \rightarrow \infty .
$$

\section{MULTIPLE BOUNDARY LAYER METHOD FOR FINITE Pr}

In this section, we will derive the lower bound of the bulk average temperature using the homogeneous ratio approach introduced by Howard ${ }^{11}$ and the multiple boundary layer method due to Busse. ${ }^{1}$ First we decompose the temperature and velocity fields into their horizontal average and fluctuating parts:

$$
T=\bar{T}+\theta, \quad \text { with } \bar{\theta}=0 \quad \text { and } \overline{\mathbf{u}}=0 \text {, }
$$




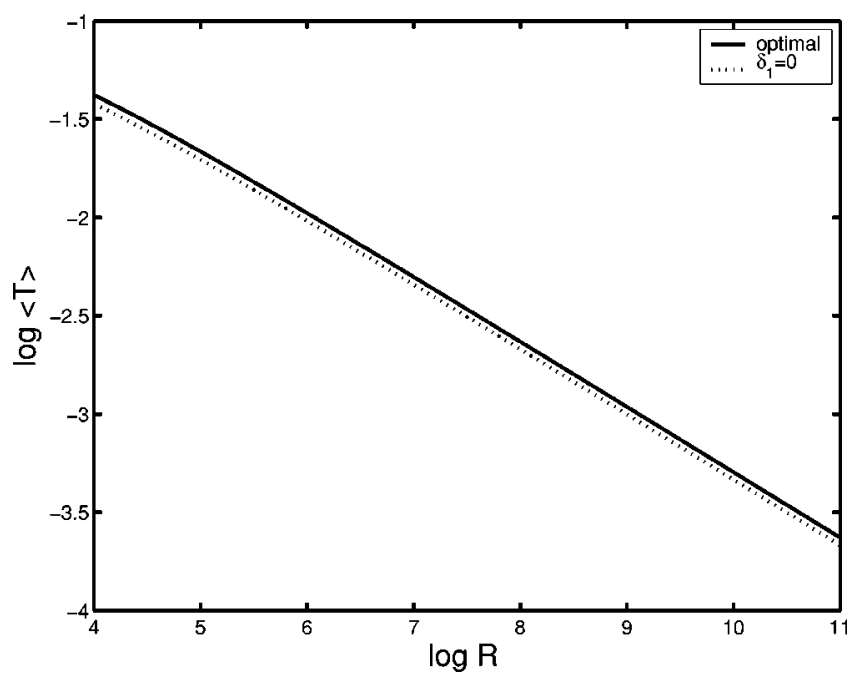

FIG. 2. The solid line is the fully optimized lower bound of the bulk average temperature compared to the estimate (30) (the dotted line).

where overline denotes the horizontal average. We will assume that the flow is statistically stationary so that the horizontal average is time-independent and the fluctuating part has vanishing horizontal mean. This is most easily justified in the limit of a horizontally infinite layer, so we take

$$
\bar{f}(z)=\lim _{L_{x}, L_{y} \rightarrow \infty} \frac{1}{L_{x} L_{y}} \int f(x, y, z, t) d x d y .
$$

The horizontal average of the temperature Eq. ( $2 b)$ is

$$
\frac{d \overline{w \theta}}{d z}=\frac{d^{2} \bar{T}}{d^{2} z}+1
$$

Integrate once to obtain

$$
\overline{w \theta}=\frac{d \bar{T}}{d z}+z+c .
$$

The integration constant $c$ here is determined by integrating above equation over [0,1], yielding

$$
\frac{d \bar{T}}{d z}=\overline{w \theta}-\langle w \theta\rangle-\left(z-\frac{1}{2}\right)
$$

Using the decomposition (34) along with (36), Eq. (2b) can be written

$$
\frac{\partial \theta}{\partial t}+w \frac{d \bar{T}}{d z}+\mathbf{u} \cdot \nabla \theta=\nabla^{2} \theta+\frac{d^{2} \bar{T}}{d^{2} z}+1=\nabla^{2} \theta+\frac{d \overline{w \theta}}{d z} .
$$

Multiplying both sides by $\theta$ and integrate over the bulk, we deduce

$$
\left\langle w \theta \frac{d \bar{T}}{d z}\right\rangle=-\left\langle|\nabla \theta|^{2}\right\rangle .
$$

Together with Eq. (38), we find the "power integral" 


$$
\left\langle\left(z-\frac{1}{2}\right) w \theta\right\rangle=\left\langle|\nabla \theta|^{2}\right\rangle+\left\langle(\overline{w \theta}-\langle w \theta\rangle)^{2}\right\rangle
$$

Another power integral is derived by multiplying Eq. ( $2 \mathrm{a})$ by $\mathbf{u}$ and integrating over the bulk:

$$
\left\langle|\nabla \mathbf{u}|^{2}\right\rangle=R\langle w \theta\rangle
$$

Finally, we derive an expression for the average temperature by multiplying Eq. (38) by $z$ and integrating over $[0,1]$ :

$$
\langle T\rangle=-\left\langle\left(z-\frac{1}{2}\right) w \theta\right\rangle+\frac{1}{12} .
$$

In summary we have the following balances:

$$
\begin{gathered}
\left\langle|\nabla \mathbf{u}|^{2}\right\rangle=R\langle w \theta\rangle, \\
\left\langle\left(z-\frac{1}{2}\right) w \theta\right\rangle=\left\langle|\nabla \theta|^{2}\right\rangle+\left\langle(\overline{w \theta}-\langle w \theta\rangle)^{2}\right\rangle, \\
\langle T\rangle=-\left\langle\left(z-\frac{1}{2}\right) w \theta\right\rangle+\frac{1}{12} .
\end{gathered}
$$

Now rewrite (45) as

$$
1=\frac{\left\langle|\nabla \theta|^{2}\right\rangle+\left\langle(\overline{w \theta}-\langle w \theta\rangle)^{2}\right\rangle}{\left\langle\left(z-\frac{1}{2}\right) w \theta\right\rangle}
$$

and multiply the $\frac{1}{12}$ in (46) so disguised to find

$$
\langle T\rangle=\frac{\left\langle|\nabla \theta|^{2}\right\rangle+\left\langle(\overline{w \theta}-\langle w \theta\rangle)^{2}\right\rangle-12\left\langle\left(z-\frac{1}{2}\right) w \theta\right\rangle^{2}}{12\left\langle\left(z-\frac{1}{2}\right) w \theta\right\rangle} .
$$

Let

$$
h(z)=\sqrt{12}\left(z-\frac{1}{12}\right) .
$$

Notice that

$$
\langle h\rangle=0, \quad\left\langle h^{2}\right\rangle=1
$$

Then

$$
\left\langle(\overline{w \theta}-h\langle h w \theta\rangle-\langle w \theta\rangle)^{2}\right\rangle=\left\langle\overline{w \theta^{2}}\right\rangle-\langle w \theta\rangle^{2}-\langle h w \theta\rangle^{2} .
$$

Thus together with (44), the average temperature can be expressed as

$$
\begin{aligned}
\langle\sqrt{12} R T\rangle & =R \frac{\left\langle|\nabla \theta|^{2}\right\rangle+\left\langle(\overline{w \theta}-\langle w \theta\rangle)^{2}\right\rangle-\langle h w \theta\rangle^{2}}{\langle h w \theta\rangle} \\
& =\frac{\left\langle|\nabla \theta|^{2}\right\rangle\left\langle|\nabla \mathbf{u}|^{2}\right\rangle}{\langle w \theta\rangle\langle h w \theta\rangle}+R \frac{\left\langle(\overline{w \theta}-h\langle h w \theta\rangle-\langle w \theta\rangle)^{2}\right\rangle}{\langle h w \theta\rangle} .
\end{aligned}
$$


The variational problem can be formulated as follows:

Given $\mu=R\langle h w \theta\rangle$, find the minimum of the functional

$$
\mathcal{F}=\frac{\left\langle|\nabla \theta|^{2}\right\rangle\left\langle|\nabla \mathbf{u}|^{2}\right\rangle}{\langle h w \theta\rangle\langle w \theta\rangle}+\mu \frac{\left\langle(\overline{w \theta}-h\langle h w \theta\rangle-\langle w \theta\rangle)^{2}\right\rangle}{\langle h w \theta\rangle^{2}}
$$

among the $\mathbf{u}, \theta$ fields with

$$
\nabla \cdot \mathbf{u}=0,\left.\quad \mathbf{u}\right|_{z=0,1}=0,\left.\quad \theta\right|_{z=0,1}=0,
$$

where

$$
w=\mathbf{u} \cdot \hat{\mathbf{k}}, \quad h(z)=\sqrt{12}\left(z-\frac{1}{2}\right) .
$$

Since the functional $\mathcal{F}$ is homogeneous in both $w$ and $\theta$, we can impose two normalization conditions

$$
\langle h w \theta\rangle=1, \quad\left\langle w^{2}\right\rangle=\left\langle\theta^{2}\right\rangle .
$$

We are seeking the minimum of the functional $\mathcal{F}$ as $\mu \rightarrow \infty$. This implies that $\overline{w \theta}=h$ $+\langle w \theta\rangle$ [here and in the following discussion the normalization conditions (56) have been assumed] throughout most of the interval $0<z<1$, which makes the second term in the functional vanish in this interval. Only near the boundary $z=0,1$ the boundary conditions prevent a close appoach of $\overline{w \theta}$ to $h+\langle w \theta\rangle$. And the contribution to the functional is thus from possible boundary layers at $z=0,1$. (Note: the boundary layers are distinct in this problem, as is the case for a similar analysis of circular Couette flow where the inner and outer cylinders must be handled seperately. ${ }^{2}$ ) Since $h(1)+\langle w \theta\rangle=\sqrt{3}+\left\langle|\nabla \mathbf{u}|^{2}\right\rangle>0$ [from Eq. (44) and definition (49)] there must be a boundary layer at $z=1$. At $z=0, h(0)+\langle w \theta\rangle=-\sqrt{3}+\langle w \theta\rangle$ is indefinite. Thus the existence of a boundary layer at $z=0$ depends on whether $h(0)+\langle w \theta\rangle$ is zero. Without loss of generality we assume there are two boundary layers at $z=0,1$ respectively, and make the ansatz

$$
w=\sum w_{n} \phi_{n}+w_{n}^{*} \phi_{n}^{*}, \quad \theta=\sum \theta_{n} \phi_{n}+\theta_{n}^{*} \phi_{n}^{*},
$$

where $\phi_{n}$ and $\phi_{n}^{*}$ satisfy

$$
\Delta_{2} \phi_{n}=-\alpha_{n}^{2} \phi_{n}, \quad \Delta_{2} \phi_{n}^{*}=-\alpha_{n}^{* 2} \phi_{n}^{*} .
$$

We introduce the following boundary layer variables:

$$
\begin{gathered}
w=\left\{\begin{array}{l}
\mu^{-p_{n}} \hat{w}\left(\zeta_{n}\right) \text { for } 1-z=O\left(\mu^{\left.-r_{n}\right),}\right. \\
\mu^{-s_{n}} \widetilde{w}\left(\zeta_{n-1}\right) \text { for } 1-z=O\left(\mu^{\left.-r_{n-1}\right),},\right.
\end{array}\right. \\
\theta= \begin{cases}\mu^{p_{n}} \hat{\theta}\left(\zeta_{n}\right) & \text { for } 1-z=O\left(\mu^{-r_{n}}\right), \\
\mu^{s_{n}} \widetilde{\theta}\left(\zeta_{n-1}\right) & \text { for } 1-z=O\left(\mu^{\left.-r_{n-1}\right),}\right.\end{cases} \\
w^{*}= \begin{cases}\mu^{-p_{n}} \hat{w}^{*}\left(\zeta_{n}^{*}\right) & \text { for } z=O\left(\mu^{\left.-r_{n}\right),}\right. \\
\mu^{-s_{n}} \widetilde{w}^{*}\left(\zeta_{n-1}^{*}\right) & \text { for } z=O\left(\mu^{-r_{n-1}}\right),\end{cases} \\
\theta^{*}= \begin{cases}\mu^{p_{n}} \hat{\theta}^{*}\left(\zeta_{n}^{*}\right) & \text { for } z=O\left(\mu^{\left.-r_{n}\right),}\right. \\
\mu^{s_{n}} \widetilde{\theta}^{*}\left(\zeta_{n-1}^{*}\right) & \text { for } z=O\left(\mu^{\left.-r_{n-1}\right),}\right.\end{cases}
\end{gathered}
$$


where

$$
\zeta_{n}=(1-z) \mu^{r_{n}}, \quad \zeta_{n}^{*}=z \mu^{r_{n}}
$$

The boundary layer structure is such that in the interior

$$
\widetilde{w}_{1} \widetilde{\theta}_{1}+\widetilde{w}_{1}^{*} \widetilde{\theta}_{1}^{*} \approx h+\langle w \theta\rangle,
$$

and in the boundary layers

$$
\widetilde{w}_{n} \widetilde{\theta}_{n}+\hat{w}_{n-1} \hat{\theta}_{n-1} \approx h_{1}+\langle w \theta\rangle, \quad \widetilde{w}_{n}^{*} \widetilde{\theta}_{n}^{*}+\hat{w}_{n-1}^{*} \hat{\theta}_{n-1}^{*} \approx h_{0}+\langle w \theta\rangle
$$

for $n=1, \ldots, N-1$, where

$$
h_{0}=h(0)=-\sqrt{3}, \quad h_{1}=h(1)=\sqrt{3} .
$$

With the boundary layer approximations, the functional becomes

$$
\begin{aligned}
\hat{\mathcal{F}}_{N}= & \frac{1}{\langle w \theta\rangle}\left\{\sum_{1}^{N} \mu^{2 p_{n}+r_{n}}\left(\int_{0}^{\infty} \hat{\theta}_{n}^{\prime 2} d \zeta_{n}+\int_{0}^{\infty} \hat{\theta}_{n}^{* \prime 2} d \zeta_{n}^{*}\right)+\sum_{2}^{N} \mu^{q_{n}-r_{n}+2 s_{n}}\left(b_{n}^{2} \int_{0}^{\infty} \widetilde{\theta}_{n}^{2} d \zeta_{n-1}\right.\right. \\
& \left.\left.+b_{n}^{* 2} \int_{0}^{\infty} \widetilde{\theta}_{n}^{* 2} d \zeta_{n-1}^{*}\right)+\mu^{q_{1}}\left(b_{1}^{2}\left\langle\widetilde{\theta}_{1}^{2}\right\rangle+b_{1}^{* 2}\left\langle\widetilde{\theta}_{1}^{* 2}\right\rangle\right)\right\}\left\{\sum _ { 1 } ^ { N } \mu ^ { 3 r _ { n } - 2 p _ { n } - q _ { n } } \left(\frac{1}{b_{n}^{2}} \int_{0}^{\infty} \hat{w}_{n}^{\prime \prime 2} d \zeta_{n}\right.\right. \\
& \left.+\frac{1}{b_{n}^{* 2}} \int_{0}^{\infty} \hat{w}_{n}^{* \prime \prime 2} d \zeta_{n}^{*}\right)+\sum_{2}^{N} \mu^{q_{n}-r_{n-1}-2 s_{n}}\left(b_{n}^{2} \int_{0}^{\infty} \widetilde{w}_{n}^{2} d \zeta_{n-1}+b_{n}^{* 2} \int_{0}^{\infty} \widetilde{w}_{n}^{* 2} d \zeta_{n-1}^{*}\right) \mu^{q_{1}}\left(b_{1}^{2}\left\langle\widetilde{w}_{1}^{2}\right\rangle\right. \\
& \left.\left.+b_{1}^{* 2}\left\langle\widetilde{w}_{1}^{* 2}\right\rangle\right)\right\}+\left\{\mu^{1-r_{N}}\left(\int_{0}^{\infty}\left(\hat{w}_{N} \hat{\theta}_{N}-h_{1}-\langle w \theta\rangle\right)^{2} d \zeta_{N}+\int_{0}^{\infty}\left(\hat{w}_{N}^{*} \hat{\theta}_{N}^{*}-h_{0}-\langle w \theta\rangle\right)^{2} d \zeta_{N}^{*}\right)\right\} .
\end{aligned}
$$

Balancing the exponents in the above expression yields

$$
r_{n}=\frac{1-4^{-n}}{3-4^{-n}}, \quad q_{n}=\frac{2-4^{-n}}{3-4^{-n}}, \quad s_{n}=0, \quad 2 p_{n}=\frac{4^{-n}}{3-4^{-n}} .
$$

Then we have

$$
\hat{\mathcal{F}}_{N}=\mu^{2 / 3-4^{-N}} F_{N},
$$

where

$$
\begin{aligned}
F_{N}= & \frac{1}{\langle w \theta\rangle}\left\{\sum_{1}^{N}\left(\int_{0}^{\infty} \hat{\theta}_{n}^{\prime 2} d \zeta_{n}+\int_{0}^{\infty} \hat{\theta}_{n}^{* \prime 2} d \zeta_{n}^{*}\right) \sum_{2}^{N}\left(b_{n}^{2} \int_{0}^{\infty} \widetilde{\theta}_{n}^{2} d \zeta_{n-1}+b_{n}^{* 2} \int_{0}^{\infty} \widetilde{\theta}_{n}^{* 2} d \zeta_{n-1}^{*}\right)+\left(b_{1}^{2}\left\langle\widetilde{\theta}_{1}^{2}\right\rangle\right.\right. \\
& \left.\left.+b_{1}^{* 2}\left\langle\widetilde{\theta}_{1}^{* 2}\right\rangle\right)\right\}\left\{\sum _ { 1 } ^ { N } ( \frac { 1 } { b _ { n } ^ { 2 } } \int _ { 0 } ^ { \infty } \hat { w } _ { n } ^ { \prime \prime 2 } d \zeta _ { n } + \frac { 1 } { b _ { n } ^ { * 2 } } \int _ { 0 } ^ { \infty } \hat { w } _ { n } ^ { * \prime 2 } d \zeta _ { n } ^ { * } ) \sum _ { 2 } ^ { N } \left(b_{n}^{2} \int_{0}^{\infty} \widetilde{w}_{n}^{2} d \zeta_{n-1}\right.\right. \\
& \left.\left.+b_{n}^{* 2} \int_{0}^{\infty} \widetilde{w}_{n}^{* 2} d \zeta_{n-1}^{*}\right)+\left(b_{1}^{2}\left\langle\widetilde{w}_{1}^{2}\right\rangle+b_{1}^{* 2}\left\langle\widetilde{w}_{1}^{* 2}\right\rangle\right)\right\}+\left\{\int_{0}^{\infty}\left(\hat{w}_{N} \hat{\theta}_{N}-h_{1}-\langle w \theta\rangle\right)^{2} d \zeta_{N}\right. \\
& \left.+\int_{0}^{\infty}\left(\hat{w}_{N}^{*} \hat{\theta}_{N}^{*}-h_{0}-\langle w \theta\rangle\right)^{2} d \zeta_{N}^{*}\right\} .
\end{aligned}
$$


Now the Euler-Lagrange equations for the functional $F_{N}$ can be written down:

$$
\begin{gathered}
\frac{1}{\langle w \theta\rangle} \frac{D_{\theta}}{b_{n}^{2}} \hat{w}_{n}^{(4)}-\mu^{r_{N}-r_{n}} \hat{\theta}_{n}\left(h_{1}+\langle w \theta\rangle-\hat{w}_{n} \hat{\theta}_{n}-\widetilde{w}_{n+1} \widetilde{\theta}_{n+1}\right)=0, \\
\frac{1}{\langle w \theta\rangle} D_{w} \hat{\theta}_{n}^{\prime \prime}+\mu^{r_{N}-r_{n}} \hat{w}_{n}\left(h_{1}+\langle w \theta\rangle-\hat{w}_{n} \hat{\theta}_{n}-\widetilde{w}_{n+1} \widetilde{\theta}_{n+1}\right)=0, \\
n=1, \ldots, N, \\
\frac{b_{n+1}^{2}}{\langle w \theta\rangle} D_{\theta} \widetilde{w}_{n+1}-\mu^{r_{N}-r_{n}} \widetilde{\theta}_{n+1}\left(h_{1}+\langle w \theta\rangle-\hat{w}_{n} \hat{\theta}_{n}-\widetilde{w}_{n+1} \widetilde{\theta}_{n+1}\right)=0, \\
\frac{b_{n+1}^{2}}{\langle w \theta\rangle} D_{w} \widetilde{\theta}_{n+1}-\mu^{r_{N}-r_{n}} \widetilde{w}_{n+1}\left(h_{1}+\langle w \theta\rangle-\hat{w}_{n} \hat{\theta}_{n}-\widetilde{w}_{n+1} \widetilde{\theta}_{n+1}\right)=0, \\
n=1, \ldots, N-1 .
\end{gathered}
$$

And for $\widetilde{w}_{1}, \widetilde{\theta}_{1}$,

$$
\begin{aligned}
& \frac{D_{\theta}}{\langle w \theta\rangle} b_{1}^{2} \widetilde{w}_{1}-\widetilde{\theta}_{1}\left\{\frac{D_{\theta} D_{w}}{2\langle w \theta\rangle^{2}}(h\langle w \theta\rangle+1)+\mu^{r_{N}}\left(h+\langle w \theta\rangle-\widetilde{w}_{1} \widetilde{\theta}_{1}-\widetilde{w}_{1}^{*} \widetilde{\theta}_{1}^{*}\right)+h\left(\int _ { 0 } ^ { \infty } \left(\hat{w}_{N} \hat{\theta}_{N}-h_{1}\right.\right.\right. \\
& \left.\left.-\langle w \theta\rangle)^{2} d \zeta_{N}+\int_{0}^{\infty}\left(\hat{w}_{N}^{*} \hat{\theta}_{N}^{*}-h_{0}-\langle w \theta\rangle\right)^{2} d \zeta_{N}^{*}\right)\right\}=0,
\end{aligned}
$$

and

$$
\begin{aligned}
& \frac{D_{w}}{\langle w \theta\rangle} b_{1}^{2} \widetilde{\theta}_{1}-\widetilde{w}_{1}\left\{\frac{D_{\theta} D_{w}}{2\langle w \theta\rangle^{2}}(h\langle w \theta\rangle+1)+\mu^{r_{N}}\left(h+\langle w \theta\rangle-\widetilde{w}_{1} \widetilde{\theta}_{1}-\widetilde{w}_{1}^{*} \widetilde{\theta}_{1}^{*}\right)+h\left(\int _ { 0 } ^ { \infty } \left(\hat{w}_{N} \hat{\theta}_{N}-h_{1}\right.\right.\right. \\
& \left.\left.\quad-\langle w \theta\rangle)^{2} d \zeta_{N}+\int_{0}^{\infty}\left(\hat{w}_{N}^{*} \hat{\theta}_{N}^{*}-h_{0}-\langle w \theta\rangle\right)^{2} d \zeta_{N}^{*}\right)\right\}=0 .
\end{aligned}
$$

The same set of equations are also satisfied by the starred quantities $\widetilde{w}_{n}^{*}, \widetilde{\theta}_{n}^{*}, \hat{w}_{n}^{*}, \hat{\theta}_{n}^{*}$.

From Eqs. (76) and (77), we have

$$
\begin{gathered}
D_{\theta} \widetilde{w}_{1}^{2}=D_{w} \widetilde{\theta}_{1}^{2}, \\
D_{\theta} \widetilde{w}_{1}^{* 2}=D_{w} \widetilde{\theta}_{1}^{* 2} .
\end{gathered}
$$

Adding these two identities yields

$$
D_{\theta}\left\langle\widetilde{w}_{1}^{2}+\widetilde{w}_{1}^{* 2}\right\rangle=D_{w}\left\langle\widetilde{\theta}_{1}^{2}+\widetilde{\theta}_{1}^{* 2}\right\rangle .
$$

Hence the normalization condition $\left\langle w^{2}\right\rangle=\left\langle\theta^{2}\right\rangle$ implies

$$
D_{\theta}=D_{w}=D \text {. }
$$

This identity together with Eqs. (76) and (77) yields

$$
\widetilde{w}_{1}^{2}=\widetilde{\theta}_{1}^{2}, \quad b_{1}=b_{1}^{*} .
$$


Equation (73) together with Eq. (75) gives

$$
D_{\theta} \widetilde{w}_{n+1}^{2}=D_{w} \widetilde{\theta}_{n+1}^{2} .
$$

The same identity holds for $\widetilde{w}_{n+1}^{*}$ and $\widetilde{\theta}_{n+1}^{*}$. Therefore

$$
\widetilde{w}_{n+1}^{2}=\widetilde{\theta}_{n+1}^{2}, \quad \widetilde{w}_{n+1}^{* 2}=\widetilde{\theta}_{n+1}^{* 2} \quad \text { for } n=1, \ldots, N-1 .
$$

Substituting the above identity back into Eq. (73), we have

$$
\begin{aligned}
& h_{1}+\langle w \theta\rangle-\hat{w}_{n} \hat{\theta}_{n}-\widetilde{w}_{n+1} \widetilde{\theta}_{n+1}=\mu^{r_{n}-r_{N}} b_{n+1}^{2} \frac{D}{\langle w \theta\rangle}, \\
& h_{0}+\langle w \theta\rangle-\hat{w}_{n}^{*} \hat{\theta}_{n}^{*}-\widetilde{w}_{n+1}^{*} \widetilde{\theta}_{n+1}^{*}=\mu^{r_{n}-r_{N}} b_{n+1}^{* 2} \frac{D}{\langle w \theta\rangle}
\end{aligned}
$$

for $n=1, \ldots, N-1$. Then Eqs. (70) and (72) become

$$
\begin{gathered}
\frac{1}{b_{n}^{2}} \hat{w}_{n}^{(4)}-b_{n+1}^{2} \hat{\theta}_{n}=0, \\
\hat{\theta}_{n}^{\prime \prime}+b_{n+1}^{2} \hat{w}_{n}=0, \quad n=1, \ldots, N-1 .
\end{gathered}
$$

The above equations hold in the region where $\hat{w}_{n} \hat{\theta}_{n} \neq h_{1}+\langle w \theta\rangle$. When the equality holds, then from Eq. (70) and Eq. (72) we can derive

$$
\frac{\hat{w}_{n}^{(4)}}{b_{n}^{2}}=-\frac{\hat{\theta}_{n} \hat{\theta}_{n}^{\prime \prime}}{w_{n}}=\left(h_{1}+\langle w \theta\rangle\right)^{2} \frac{\hat{w}_{n}^{\prime \prime} \hat{w}_{n}-2 \hat{w}_{n}^{\prime 2}}{\hat{w}_{n}^{5}} .
$$

With the following change of variables,

$$
\left\{\begin{array}{l}
\zeta=b_{n}^{1 / 3} b_{n+1}^{2 / 3} \zeta_{n}, \\
\hat{\Omega}=b_{n}^{-1 / 3} b_{n+1}^{1 / 3}\left(h_{1}+\langle h w \theta\rangle\right)^{-1 / 2} \hat{w}_{n}, \\
\hat{\Theta}=b_{n}^{1 / 3} b_{n+1}^{-1 / 3}\left(h_{1}+\langle h w \theta\rangle\right)^{-1 / 2} \hat{\theta}_{n},
\end{array}\right.
$$

Eqs. (86), (87), and (88) become

$$
\left\{\begin{array}{l}
\hat{\Omega}^{(4)}-\hat{\Theta}=0, \\
\hat{\Theta}^{\prime \prime}+\hat{\Omega}=0, \\
\hat{\Omega}^{(4)}=\frac{\hat{\Omega}^{\prime \prime} \hat{\Omega}-2 \hat{\Omega}^{\prime 2}}{\hat{\Omega}^{5}} .
\end{array}\right.
$$

Starred quantities satisfy the same equations with $h_{1}$ replaced by $h_{0}$. This set of differential equations has been studied in Ref. 1 , where the constant $\beta$ is defined

$$
3 \beta=\int_{0}^{\infty} \hat{\Omega}^{\prime \prime 2} d \zeta+\int_{0}^{\infty}(1-\hat{\Omega} \hat{\Theta}) d \zeta=1.847
$$

and the following integrals are evaluated: 


$$
\int_{0}^{\infty} \frac{w_{n}^{\prime \prime 2}}{b_{n}^{2}} d \zeta_{n}+\int_{0}^{\infty} b_{n+1}^{2} \widetilde{w}_{n+1}^{2} d \zeta_{n}=3 \beta\left(h_{1}+\langle w \theta\rangle\right) b_{n}^{-1 / 3} b_{n+1}^{4 / 3}, \quad n=1, \ldots, N-1
$$

When $n=N$, the differential equations for $\hat{w}_{N}$ and $\hat{\theta}_{N}$ are

$$
\begin{gathered}
\frac{D}{\langle w \theta\rangle b_{N}^{2}} \hat{w}_{N}^{(4)}-\left(h_{1}+\langle w \theta\rangle-\hat{w}_{N} \hat{\theta}_{N}\right) \hat{\theta}_{N}=0, \\
\frac{D}{\langle w \theta\rangle b_{N}^{2}} \hat{\theta}_{N}^{\prime \prime}+\left(h_{1}+\langle w \theta\rangle-\hat{w}_{N} \hat{\theta}_{N}\right) \hat{w}_{N}=0 .
\end{gathered}
$$

Then with the following change of variables,

$$
\left\{\begin{array}{l}
\zeta=b_{N}^{1 / 3}\left(h_{1}+\langle w \theta\rangle\right)^{1 / 3}\left(\frac{D}{\langle w \theta\rangle}\right)^{-1 / 3} \zeta_{N}, \\
\Omega=b_{N}^{-1 / 3}\left(h_{1}+\langle h w \theta\rangle\right)^{-1 / 3}\left(\frac{D}{\langle w \theta\rangle}\right)^{-1 / 6} \hat{w}_{N} \\
\Theta=b_{N}^{1 / 3}\left(h_{1}+\langle h w \theta\rangle\right)^{-2 / 3}\left(\frac{D}{\langle w \theta\rangle}\right)^{1 / 6} \hat{\theta}_{N}
\end{array}\right.
$$

Eqs. (93) and (94) become

$$
\begin{gathered}
\Omega^{(4)}-(1-\Omega \Theta) \Theta=0, \\
\Theta^{\prime \prime}+(1-\Omega \Theta) \Omega=0 .
\end{gathered}
$$

In Howard's paper ${ }^{11}$ the following result is given:

$$
\sigma=\int_{0}^{\infty} \Omega^{\prime \prime 2} d \zeta=\int_{0}^{\infty} \Theta^{\prime 2} d \zeta=\frac{1}{4} \int_{0}^{\infty}(1-\Omega \Theta)^{2} d \zeta=0.337 .
$$

Thus the following integrals can be expressed in $\sigma$ :

$$
\begin{gathered}
\int_{0}^{\infty} \frac{\left(\hat{w}^{(4)}\right)^{2}}{b_{N}^{2}} d \zeta_{N}=\sigma\left(h_{1}+\langle w \theta\rangle\right)^{5 / 3}\left(\frac{D}{\langle w \theta\rangle}\right)^{-2 / 3} b_{N}^{-1 / 3}, \\
\int_{0}^{\infty} \hat{\theta}_{N}^{\prime 2} d \zeta_{N}=\sigma\left(h_{1}+\langle w \theta\rangle\right)^{5 / 3}\left(\frac{D}{\langle w \theta\rangle}\right)^{-2 / 3} b_{N}^{-1 / 3}, \\
\int_{0}^{\infty}\left(h_{1}+\langle h w \theta\rangle-\hat{w}_{N} \hat{\theta}_{N}\right)^{2} d \zeta_{N}=4 \sigma\left(h_{1}+\langle w \theta\rangle\right)^{5 / 3}\left(\frac{D}{\langle w \theta\rangle}\right)^{1 / 3} b_{N}^{-1 / 3} .
\end{gathered}
$$

Putting the above integrals together, the functional $F_{N}$ can then be expressed as

$$
\begin{aligned}
F_{N}= & \frac{D^{2}}{\langle w \theta\rangle} \\
& +4 \sigma\left(h_{1}+\langle w \theta\rangle\right)^{5 / 3}\left(\frac{D}{\langle w \theta\rangle}\right)^{1 / 3} b_{N}^{-1 / 3}+4 \sigma\left(h_{0}+\langle w \theta\rangle\right)^{5 / 3}\left(\frac{D}{\langle w \theta\rangle}\right)^{1 / 3} b_{N}^{*-1 / 3},
\end{aligned}
$$

and 


$$
\begin{aligned}
D= & \sum_{n=1}^{N-1} 3 \beta\left\{\left[\frac{b_{n+1}^{4}}{b_{n}}\right]^{1 / 3}\left(h_{1}+\langle w \theta\rangle\right)+\left[\frac{b_{n+1}^{* 4}}{b_{n}^{*}}\right]^{1 / 3}\left(h_{0}+\langle w \theta\rangle\right)\right\}+\sigma\left(\frac{D}{\langle w \theta\rangle}\right)^{-2 / 3}\left\{\left(h_{1}+\langle w \theta\rangle\right)^{5 / 3} b_{N}^{-1 / 3}\right. \\
& \left.+\left(h_{0}+\langle w \theta\rangle\right)^{5 / 3} b_{N}^{*-1 / 3}\right\}+b_{1}^{2}\langle w \theta\rangle .
\end{aligned}
$$

Minimizing $F_{N}$ with respect to $b_{n}$ and $b_{n}^{*}$ yields

$$
\begin{aligned}
\frac{\partial D}{\partial b_{1}} & =0 \Rightarrow 2 b_{1}\langle w \theta\rangle=\beta\left[\left(h_{1}+\langle w \theta\rangle\right)\left(\frac{b_{2}}{b_{1}}\right)^{4 / 3}+\left(h_{0}+\langle w \theta\rangle\right)\left(\frac{b_{2}^{*}}{b_{1}}\right)^{4 / 3}\right], \\
\frac{\partial D}{\partial b_{n}} & =0 \Rightarrow\left[\frac{b_{n+1}}{b_{n}}\right]^{4 / 3}=4\left[\frac{b_{n}}{b_{n-1}}\right]^{1 / 3}, \\
\frac{\partial D}{\partial b_{n}^{*}} & =0 \Rightarrow\left[\frac{b_{n+1}^{*}}{b_{n}^{*}}\right]^{4 / 3}=4\left[\frac{b_{n}^{*}}{b_{n-1}^{*}}\right]^{1 / 3}, \\
\frac{\partial F_{N}}{\partial b_{N}} & =0 \Rightarrow\left[\frac{b_{N+1}}{b_{N}}\right]^{4 / 3}=4\left[\frac{b_{N}}{b_{N-1}}\right]^{1 / 3}, \\
\frac{\partial D}{\partial b_{N}^{*}} & =0 \Rightarrow\left[\frac{b_{N+1}^{*}}{b_{N}^{*}}\right]^{4 / 3}=4\left[\frac{b_{N}^{*}}{b_{N-1}^{*}}\right]^{1 / 3},
\end{aligned}
$$

where

$$
\begin{aligned}
& b_{N+1}=\left(\frac{\sigma}{\beta}\right)^{4 / 3}\left(\frac{\left(h_{1}+\langle w \theta\rangle\right)\langle w \theta\rangle}{D}\right)^{1 / 2}, \\
& b_{N+1}^{*}=\left(\frac{\sigma}{\beta}\right)^{4 / 3}\left(\frac{\left(h_{0}+\langle w \theta\rangle\right)\langle w \theta\rangle}{D}\right)^{1 / 2} .
\end{aligned}
$$

From the above relations, the $b_{n}$ can be determined:

$$
b_{n+1}=4^{n-1}\left[\left(\frac{b_{N+1}}{4^{N-1}}\right)^{1-4^{-n}} \cdot\left(4 b_{1}\right)^{4^{-n}-4^{-N}}\right]^{1 / 1-4^{-N}} .
$$

And $b_{n+1}^{*}$ has a similar form:

$$
b_{n+1}^{*}=4^{n-1}\left[\left(\frac{b_{N+1}^{*}}{4^{N-1}}\right)^{1-4^{-n}} \cdot\left(4 b_{1}\right)^{4^{-n}-4^{-N}}\right]^{1 / 1-4^{-N}} .
$$

It is clear from the above expressions that $b_{n} \neq b_{n}^{*}$ for $n \neq 1$ since $b_{N}$ [Eq. (110)] is different from $b_{N}^{*}$ [Eq. (111)]. Finally, $b_{1}$ can be solved from (106) and the recursion relation

$$
\begin{aligned}
b_{1}= & \left\{\frac { \beta } { 2 ^ { 5 / 3 } \langle w \theta \rangle } ( \frac { \sigma } { \beta } ) ^ { 3 / 4 ( 1 - 4 ^ { - N } ) } \left[\left(h_{1}+\langle w \theta\rangle\right)^{4 /\left(1-4^{-N}\right) / 3-4^{-N}}\right.\right. \\
& \left.\left.+\left(h_{0}+\langle w \theta\rangle\right)^{4 /\left(1-4^{-N}\right) / 3-4^{-N}}\right]\right\}
\end{aligned}
$$

Putting all these together, the prefactor $F_{N}$ is a function of $\langle w \theta\rangle$ only: 


$$
\begin{aligned}
F_{N}= & \frac{D^{2}}{\langle w \theta\rangle} \frac{3-4^{-N}}{1-4^{-N}} \\
= & \left(3-4^{-N}\right)\left(1-4^{-N}\right) 2^{-4 N 4^{-N} / 3-4^{-N}} \cdot\left(2^{5 / 3} \beta \times\left(\frac{\sigma}{\beta}\right)^{3 / 4\left(1-4^{-N}\right)}\right)^{4\left(1-4^{-N}\right) / 3-4^{-N}} \\
& \times\left[\frac{\left(h_{1}+\langle w \theta\rangle\right)^{3-2 \cdot 4^{-N} / 2\left(1-4^{-N}\right)}+\left(h_{0}+\langle w \theta\rangle\right)^{3-2 \cdot 4^{-N} / 2\left(1-4^{-N}\right)}}{\langle w \theta\rangle^{1-3 \cdot 4^{-N} / 4\left(1-4^{-N}\right)}}\right]^{4\left(1-4^{-N}\right) / 3-4^{-N}} .
\end{aligned}
$$

Now the value of $\langle w \theta\rangle$ can be determined by setting $d F_{N} / d\langle w \theta\rangle$ to zero. The resulting equation for $\langle w \theta\rangle$ is

$$
(\alpha-1) x^{(3-2 c) / 2(1-c)}-\alpha x-\alpha x^{1 /(1-c)}+(\alpha-1)=0,
$$

where

$$
x=\frac{\sqrt{3}+\langle w \theta\rangle}{\sqrt{3}-\langle w \theta\rangle}, \quad \alpha=\frac{3-2 c}{1-3 c}, \quad c=4^{-N} .
$$

For general values of $N$, the above equation has to be solved numerically:

$$
\begin{aligned}
& N=1, \quad\langle w \theta\rangle=0.4831, \\
& N=2, \quad\langle w \theta\rangle=0.9259, \\
& N=3, \quad\langle w \theta\rangle=1.0120,
\end{aligned}
$$

When $N \rightarrow \infty$, the above equation can be solved exactly:

$$
\langle w \theta\rangle_{\infty}=\frac{3 \sqrt{3}}{5}=1.039 .
$$

This shows that there indeed is a boundary layer at $z=0$ since all $\langle w \theta\rangle^{\prime}$ s are less than $h_{0}=\sqrt{3}$.

Now we can write down the scaling of $\langle T\rangle$ as $N \rightarrow \infty$ :

$$
\langle T\rangle=\frac{1}{\sqrt{12} R} F_{\infty} \mu^{2 / 3}=10.285 \mu^{2 / 3} R^{-1} .
$$

Recalling the identity (46):

$$
\langle T\rangle=-\left\langle\left(z-\frac{1}{2}\right) w \theta\right\rangle+\frac{1}{12}
$$

we know that as $\mu \rightarrow \infty$

$$
\mu \sim \frac{1}{\sqrt{12}} R
$$

This leads to the scaling bound on $\langle T\rangle$ with respect to $R$ :

$$
\langle T\rangle \geqslant 4.421 R^{-1 / 3} \text {. }
$$


The profiles of $\widetilde{w}_{1}$ and $\widetilde{\theta}_{1}$ can be determined from the fact that in the interior of the interval $0<z<1$,

$$
\widetilde{w}_{1} \widetilde{\theta}_{1} \approx h+\langle w \theta\rangle, \text { and } \quad \widetilde{w}_{1}=\widetilde{\theta}_{1} .
$$

In the case $N \rightarrow \infty, h=2 \sqrt{3} z-(2 \sqrt{3} / 5)$. And then

$$
\tilde{w}_{1}=\sqrt{2 \sqrt{3} z-\frac{2 \sqrt{3}}{5} \mid}, \quad \tilde{\theta}_{1}= \pm \sqrt{2 \sqrt{3} z-\frac{2 \sqrt{3}}{5} \mid} .
$$

However, whether $\theta$ changes sign in $0<z<1$ can not be inferred from the variational problem since only the product of $w$ and $\theta$ appears in the functional $\mathcal{F}$. Thus the possibility of $w$ changing its sign cannot be excluded.

\section{BACKGROUND METHOD FOR INFINITE Pr}

As can be seen from the momentum equation (2a), the velocity field is instantaneously slaved to the temperature field in the limit $\operatorname{Pr} \rightarrow \infty$. Then it is straightforward to extract the equation satisfied by the vertical velocity $w$ for a given fluctuation field $\theta$ :

$$
\Delta^{2} w=-R \Delta_{H} \theta
$$

The incompressibility condition on the velocity field combined with the no-slip boundary conditions at $z=0$ and $z=1$ imply that both $w$ and $\partial w / \partial z$ vanish at the rigid boundaries. To implement the background analysis we decompose the temperature field as we did for the finite Pr case and notice that identies (10) and (14) still hold. This observation leads to the bound (18):

$$
\langle T\rangle \geqslant 2\langle\tau\rangle-\left\langle\tau^{\prime 2}\right\rangle
$$

provided the functional (17)

$$
H=\left\langle|\nabla \theta|^{2}\right\rangle+\left\langle\left(2 \tau^{\prime}-a\right) w \theta\right\rangle+\frac{a}{R}\left\langle|\nabla \mathbf{u}|^{2}\right\rangle
$$

is positive semidefinite among divergence free velocity fields satisfying Eq. (123) and no-slip boundary conditions at $z=0,1$, and temperature fields $\theta(x, y, z, t)$ vanishing at $z=0,1$. The constraint on the background field $\tau(z)$ is the same: $\tau(0)=\tau(1)=0$.

It is convenient to find the sufficient conditions for the non-negativity of $H$ in its Fourier series representation $H=\Sigma_{\mathbf{k}} H_{\mathbf{k}}$, where

$$
\begin{aligned}
H_{\mathbf{k}}\left\{\theta_{\mathbf{k}}\right\}= & \int_{0}^{1}\left[\left|D \theta_{\mathbf{k}}\right|^{2}+k^{2}\left|\theta_{\mathbf{k}}\right|^{2}+\left(\tau^{\prime}-\frac{a}{2}\right)\left(w_{\mathbf{k}}^{*} \theta_{\mathbf{k}}+w_{\mathbf{k}} \theta_{\mathbf{k}}^{*}\right)+\frac{a}{R}\left(\frac{1}{k^{2}}\left|D^{2} w_{\mathbf{k}}\right|^{2}+2\left|D w_{\mathbf{k}}\right|^{2}\right.\right. \\
& \left.\left.+k^{2}\left|w_{\mathbf{k}}\right|^{2}\right)\right] d z,
\end{aligned}
$$

where $w_{\mathbf{k}}(z)$ and $\theta_{\mathbf{k}}(z)$ are the Fourier components of $w$ and $\theta$ corresponding to wave number $\mathbf{k}$, satisfying

$$
\left(-D^{2}+k^{2}\right)^{2} w_{\mathbf{k}}=R k^{2} \theta_{\mathbf{k}}
$$

Then $H \geqslant 0$ iff each $H_{\mathbf{k}}$ is positive semidefinite for complex valued functions $\theta_{\mathbf{k}}$ of a single (real) variable $z$ where $w_{\mathbf{k}}$ solves the fourth-order linear boundary value problem above with both homogeneous Dirichlet and Neumann boundary conditions on [0,1]. 


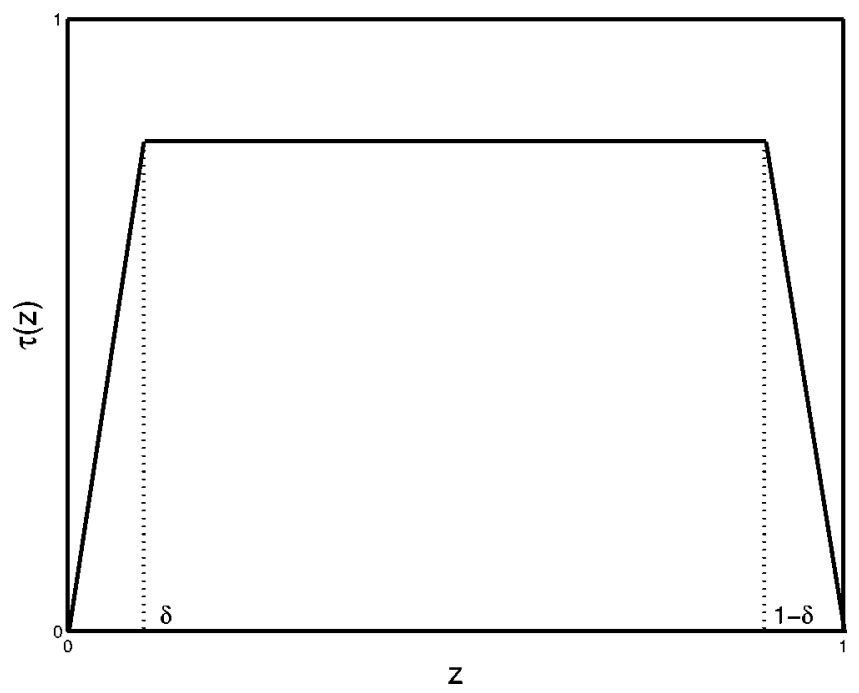

FIG. 3. The background profile for infinite Prandtl number.

We need to choose the background field $\tau(z)$ before we can estimate the magnitude of $H_{\mathbf{k}}$. Since the expressions for the lower bound (18) and the functional $H(17)$ have the same forms as in the finite Pr case, we can choose the same background profile (19). And consequently, after maximizing the bound over $a$ and $b$, we arrive at the same expression (21):

$$
\langle T\rangle \geqslant \frac{1}{4}\left(1-\delta_{1}\right)\left(1-\delta_{2}\right)\left(\delta_{1}+\delta_{2}\right)
$$

This expression is invariant if we exchange $\delta_{1}$ and $\delta_{2}$, and the estimate (132) is pointwise. This suggests that the maximum of the right-hand side of (21) occurs when $\delta_{1}=\delta_{2}=\delta$. And then

$$
\left\{\begin{array}{l}
a=0, \\
b=\frac{\delta(1-\delta)}{2}
\end{array}\right.
$$

by Eq. (22). The background profile becomes

$$
\tau(z)=\left\{\begin{array}{l}
\frac{1-\delta}{2} z, \quad 0 \leqslant z<\delta, \\
\frac{\delta(1-\delta)}{2}, \quad 1-\delta \leqslant z \leqslant 1-\delta, \\
\frac{1-\delta}{2}(1-z), \quad 1-\delta \leqslant z \leqslant 1,
\end{array}\right.
$$

and the bound is

$$
\langle T\rangle \geqslant \frac{\delta(1-\delta)^{2}}{2}
$$

as long as $\delta$ is chosen to ensure $H$ is semipositive definite (see Fig. 3).

In the following we will use the inequality, proved in Ref. 10, for solutions of (127): 


$$
\left|w_{\mathbf{k}}\right| \leqslant \frac{1}{2} z^{2} \frac{R}{\sqrt{C}} k\left\|\theta_{\mathbf{k}}\right\|
$$

for $z \in\left[0, \frac{1}{2}\right]$, where $C=\frac{1}{2}(7-\sqrt{41})$. (A similar estimate holds on the other end of the unit interval.) Applying this estimate, we have

$$
\begin{aligned}
& \left|\int_{0}^{1}\left(\tau^{\prime}-\frac{a}{2}\right)\left(w_{\mathbf{k}}^{*} \theta_{\mathbf{k}}+w_{\mathbf{k}} \theta_{\mathbf{k}}^{*}\right) d z\right| \\
& =\left|\int_{0}^{1} \tau^{\prime}\left(w_{\mathbf{k}}^{*} \theta_{\mathbf{k}}+w_{\mathbf{k}} \theta_{\mathbf{k}}^{*}\right) d z\right| \\
& \leqslant 2 \frac{1-\delta}{2} \int_{0}^{\delta}\left|w_{\mathbf{k}}\right|\left|\theta_{\mathbf{k}}\right| d z+2 \frac{1-\delta}{2} \int_{1-\delta}^{1}\left|w_{\mathbf{k}}\right|\left|\theta_{\mathbf{k}}\right| d z \\
& \leqslant(1-\delta) \int_{0}^{\delta} \frac{1}{2} z^{2}\left(\frac{R}{\sqrt{C}} k\left\|\theta_{\mathbf{k}}\right\|\right) \sqrt{z \int_{0}^{1 / 2}\left|D \theta_{\mathbf{k}}\left(z^{\prime}\right)\right|^{2} d z^{\prime}} d z+(1-\delta) \int_{1-\delta}^{1} \frac{1}{2}(1-z)^{2} \\
& \times\left(\frac{R}{\sqrt{C}} k\left\|\theta_{\mathbf{k}}\right\|\right) \times \sqrt{(1-z) \int_{1 / 2}^{1}\left|D \theta_{\mathbf{k}}\left(z^{\prime}\right)\right|^{2} d z^{\prime}} d z \\
& \leqslant(1-\delta) \frac{R}{\sqrt{C}} k\left\|\theta_{\mathbf{k}}\right\| \frac{1}{7} \delta_{1}^{7 / 2} \sqrt{\int_{0}^{1 / 2}\left|D \theta_{\mathbf{k}}\left(z^{\prime}\right)\right|^{2} d z^{\prime}} \\
& +(1-\delta) \frac{R}{\sqrt{C}} k\left\|\theta_{\mathbf{k}}\right\| \frac{1}{7} \delta_{1}^{7 / 2} \sqrt{\int_{1 / 2}^{1}\left|D \theta_{\mathbf{k}}\left(z^{\prime}\right)\right|^{2} d z^{\prime}} \\
& \leqslant(1-\delta) \frac{R}{\sqrt{C}} \frac{\delta^{7 / 2}}{7} \frac{k}{2}\left(\frac{k\left\|\theta_{\mathbf{k}}\right\|^{2}}{\sqrt{2}}+\frac{\sqrt{2}}{k} \int_{0}^{1 / 2}\left|D \theta_{\mathbf{k}}\left(z^{\prime}\right)\right|^{2} d z^{\prime}\right) \\
& +(1-\delta) \frac{R}{\sqrt{C}} \frac{\delta^{7 / 2}}{7} \frac{k}{2}\left(\frac{k\left\|\theta_{\mathbf{k}}\right\|^{2}}{\sqrt{2}}+\frac{\sqrt{2}}{k} \int_{0}^{1 / 2}\left|D \theta_{\mathbf{k}}\left(z^{\prime}\right)\right|^{2} d z^{\prime}\right) \\
& =(1-\delta) \frac{R}{\sqrt{C}} \frac{1}{7} \delta^{7 / 2} \frac{1}{\sqrt{2}}\left(k^{2}\left\|\theta_{\mathbf{k}}\right\|^{2}+\left\|D \theta_{\mathbf{k}}\right\|^{2}\right) .
\end{aligned}
$$

Then

$$
H \geqslant k^{2}\left(1-\frac{(1-\delta) \delta^{7 / 2}}{7 \sqrt{2}} \frac{R}{\sqrt{C}}\right)\left(k^{2}\left\|\theta_{\mathbf{k}}\right\|^{2}+\left\|D \theta_{\mathbf{k}}\right\|^{2}\right) .
$$

Choosing $\delta$ such that

$$
\frac{(1-\delta) \delta^{7 / 2}}{14} \frac{R}{\sqrt{C}}=1,
$$

the non-negativity of $H$ is ensured. Then as $R \rightarrow \infty, \delta \sim(7 \sqrt{2 C} / R)^{2 / 7}$ and thus

$$
\langle T\rangle \geqslant \frac{1}{2} \delta=\frac{1}{2}\left(\frac{7 \sqrt{2 C}}{R}\right)^{2 / 7} .
$$




\section{SUMMARY AND DISCUSSION}

The preceeding sections we have proven that for the finite (or arbitrary) Prandtl number case, in nondimensional units,

$$
\langle T\rangle \geqslant c_{1} R^{-1 / 3},
$$

and for the infinite Prandtl number model,

$$
\langle T\rangle \geqslant c_{2} R^{-2 / 7} .
$$

In dimensional units of temperature and heat flux these results are

$$
\langle T\rangle \geqslant \widetilde{c}_{1} H^{2 / 3}
$$

for arbitrary Pr (see Ref. 4 for a similar estimate in that case of an internally heated self-gravitating sphere), and

$$
\langle T\rangle \geqslant \widetilde{c}_{2} H^{5 / 7}
$$

for the infinite Pr.

Recent numerical experiments ${ }^{18}$ on thermal convection with internal heating in a fluid layer with infinite Prandtl number suggest that

$$
\langle T\rangle \propto R^{-0.234} .
$$

The observed exponent 0.234 is smaller than the rigorous estimate derived here, $2 / 7 \approx 0.286$, but consistent with the bound. In the case of Rayleigh-Bénard convection, the methods employed here produce scaling (upper) bounds on the heat transport ${ }^{1,9,10}$ that are also consistent—but not in total agreement-with observed high Rayleigh number scalings.

It is worthwhile to note that the "optimal" background profile that the analysis suggests (Fig. 1 ) is suggestive of the mean temperature profile one expects for the internal heating problem. That is, the buoyancy force driving the convection will concentrate the warmer fluid near the top of the layer. Interestingly, this is not the case for the infinite Pr problem where the "optimal" temperature background maintains the symmetry of the conduction solution. It remains an open problem to apply the multiple boundary layer analysis to the case of infinite $\mathrm{Pr}$, as it has previously been applied for the case of Rayleigh-Bénard convection. ${ }^{5}$ A full (numerical) solution of the optimal background variational problem, as has recently been accomplished for Rayleigh-Bénard convection with finite Prandtl number, ${ }^{15}$ could improve the estimates further.

\section{ACKNOWLEDGMENTS}

This research was initiated while the authors were participants in the 2002 Geophysical Fluid Dynamics (GFD) Program at Woods Hole Oceanographic Institution. We are grateful to that staff and Fellows of the GFD program for helpful discussions. Two of us (L.L. and C.R.D.) thank Professor C. R. Lithgow-Bertelloni for her suggestions and encouragement. This work was supported in part by NSF Awards PHY-9900635 and PHY-0244859.

\footnotetext{
${ }^{1}$ Busse, F. H., "On Howard's upper bound for heat transport by turbulent convection,” J. Fluid Mech. 37, 457-477 (1969).

${ }^{2}$ Busse, F., "The bounding theory of turbulence and its physical significance in the case of turbulent Couette flow," in Statistical Models and Turbulence, edited by M. Rosenlatt and C. M. Van Atta, Springer Lecture Notes in Physics Vol. 12 (Springer, Berlin, 1972), pp. 103-126.

${ }^{3}$ Busse, F., "The optimum theory of turbulence," Adv. Appl. Mech. 18, 77-121 (1978).

${ }^{4}$ Busse, F., On the Optimum Theory of Turbulence, Energy Stability and Convection, Pitman Research Notes in Mathematics, edited by G. Galdi and B. Straughan (Wiley, New York, 1987).

${ }^{5}$ Chan, S.K., "Infinite Prandtl number turbulent convection," Stud. Appl. Math. 50, 13-49 (1971).
} 
${ }^{6}$ Constantin, P. and Doering, C. R., "Infinite Prandtl number convection,” J. Stat. Phys. 94, 159-172 (1999).

${ }^{7}$ G. F. Davies, Dynamic Earth: Plates, Plumes, and Mantle Convection (Cambridge University Press, New York, 1999).

${ }^{8}$ Doering, C. R., and Constantin, P., "Energy dissipation in shear driven turbulence," Phys. Rev. Lett. 69, 1648-1651 (1992).

${ }^{9}$ Doering, C. R., and Constantin, P., "Variational bounds on energy dissipation in incompressible flows. III. Convection," Phys. Rev. E 53, 5957-5981 (1996).

${ }^{10}$ Doering, C. R., and Constantin, P., "On upper bounds for infinite Prandtl number convection," J. Math. Phys. 42, 784-795 (2001).

${ }^{11}$ Howard, L. N., "Heat transport by turbulent convection," J. Fluid Mech. 17, 405-432 (1963).

${ }^{12}$ Howard, L. N., "Bounds on flow quantities," Annu. Rev. Fluid Mech. 4, 473-494 (1972).

${ }^{13}$ Nicodemus, R., Grossmann, S., and Holthaus, M., "Improved variational principle for bounds on energy dissipation in turbulent shear flow," Physica D 101, 178-190 (1997).

${ }^{14}$ Otero, J., Wittenberg, R. W., Worthing, R. A., and Doering, C. R., "Bounds on Rayleigh-Bénard convection with an imposed heat flux," J. Fluid Mech. 473, 191-199 (2002).

${ }^{15}$ Plasting, S. C., and Kerswell, R. R., "Improved upper bound on the energy dissipation rate in plane Couette flow: The full solution to Busse's problem and the Constantin-Doering-Hopf problem with one-dimensional background field," J. Fluid Mech. 477, 363-379 (2003).

${ }^{16}$ Roberts, P. H., "Convection in horizontal layers with internal heating: Theory," J. Fluid Mech. 30, 33-49 (1967).

${ }^{17}$ Schubert, G., Turcotte, D. L., and Olson, P., Mantle Convection in the Earth and Planets (Cambridge University Press, New York, 2001).

${ }^{18}$ Sotin, C., and Labrosse, S., "Three-dimensional thermal convection in an iso-viscous, infinite Prandtl number fluid heated from within and from below: Applications to the transfer of heat through planetary mantles," Phys. Earth Planet. Inter. 112, 171-190 (1999).

${ }^{19}$ Sparrow, E. M., Goldstein, R. J., and Jonsson, V. K., "Thermal instability in a horizontal fluid layer: Effect of boundary conditions and nonlinear temperature profile," J. Fluid Mech. 18, 513-528 (1964). 